\title{
HIGH GENETIC MACROLIDE RESISTANCE IN DIFFERENT STRAIN TYPES OF TREPONEMA PALLIDUM IN BARCELONA
}

Fernández-Naval $\mathbf{C}^{1,2,3}$, Arando $\mathrm{M}^{3,4}$, Antón $\mathrm{A}^{2,3}$, González-López $\mathrm{JJ}^{1,2,3}$, Espasa $\mathrm{M}^{2}$, Fernández-Huerta $\mathrm{M}^{2}$, Andrés $\mathbf{C}^{1,2,3}$ Piñana $\mathbf{M}^{1,2,3}$, Serra-Pladevall $\mathrm{J}^{1,2,3}$, Pumarola $\mathrm{T}^{1,2,3}$, Vall-Mayans $\mathbf{M}^{4}$, Esperalba $\mathrm{J}^{2}$

1 Vall d'Hebron Research Institute (VHIR), Barcelona, Spain, ${ }^{2}$ Microbiology Department, Hospital Universitari Vall d'Hebron (HUVH), Barcelona, Spain ${ }^{3}$ Universitat Autònoma de Barcelona (UAB), Barcelona, Spain ${ }^{4}$ STI Unit Vall d'Hebron-Drassanes, Hospital Universitari Vall d'Hebron, Barcelona, Spain

\section{BACKGROUND}

The distribution of macrolide-resistant Treponema pallidum subsp. pallidum ( $T$. pallidum) is widespread in some countries despite being the second line treatment for syphilis. The A2058G and A2059G mutations in the 23S rRNA gene are mostly associated with macrolide resistance as in other sexually transmitted infections (STIs).

The aim of this study is to describe the prevalence of the mutations conferring resistance to macrolides in different T.pallidum strain-types from a cohort of patients diagnosed with syphilis in Barcelona, Spain.

\section{MATERIAL AND METHODS}

A total of 99 swabs from genital ulcer and 120 whole blood samples were collected from 183 subjects diagnosed with primary or secondary syphilis at the STI Unit Vall d'HebronDrassanes (Barcelona, Spain) during 2015. T. pallidum was detected by a specific tpp47 gene assay. The strain-types were previously identified in positive samples following the Enhanced CDC Typing methodology based on the analysis of the arp, tpr and tp0548 genes. The identification of A2058G and A2059G mutations in the 23S rRNA conferring macrolide resistance was performed by Sanger sequencing of both copies of the gene.

\section{RESULTS}

T. pallidum was detected in $130 / 219$ (59\%) of the samples collected. From the positive samples, 62/130 (48\%) were fullytyped and 44/130 (34\%) were partially-typed (at least by one of the ECDCT genes). The 23S rRNA gene was sequenced in $118 / 130$ (91\%) samples (at least by one copy) (table 1).

Table 1. Number of samples harbouring the macrolide resistance mutations

\begin{tabular}{ccccc}
\hline \multirow{2}{*}{$\begin{array}{c}\text { Copies } \\
\text { sequenced }\end{array}$} & $\begin{array}{c}\text { Positive } \\
\text { samples }\end{array}$ & \multicolumn{3}{c}{ 23S rRNA variant } \\
\cline { 3 - 5 } 2 copies & 99 & 6 & 92 & 1 \\
$\mathbf{1}$ copy & 19 & 0 & 19 & 0 \\
$\mathbf{0}$ copies & 12 & 0 & 0 & 0 \\
\hline Total & 130 & 6 & 111 & 1 \\
\hline
\end{tabular}

Up to $112 / 118$ (95\%) samples harboured one of the two mutations associated with macrolide resistance. The A2058G mutation was found in $111 / 112$ (98\%) cases while the mutation at the gene position 2059 was found just in one case (1/112; $0.9 \%$ ). Associations with the type of specimen (swab or whole blood) or the syphilis stage (primary and secondary) could not be found. All the samples typed as $14 \mathrm{~d} / \mathrm{g}$ and $14 \mathrm{f} / \mathrm{g}$ (the most frequent types) carried the A2058G mutation (figure 1). The samples with non-mutated 23S rRNA gene $(n=5)$ were distributed in three infrequent strain-types (14d/f: 3/5; 14f/f: 1/3; 14j/d: 1/1).

\section{Vall d'Hebron}

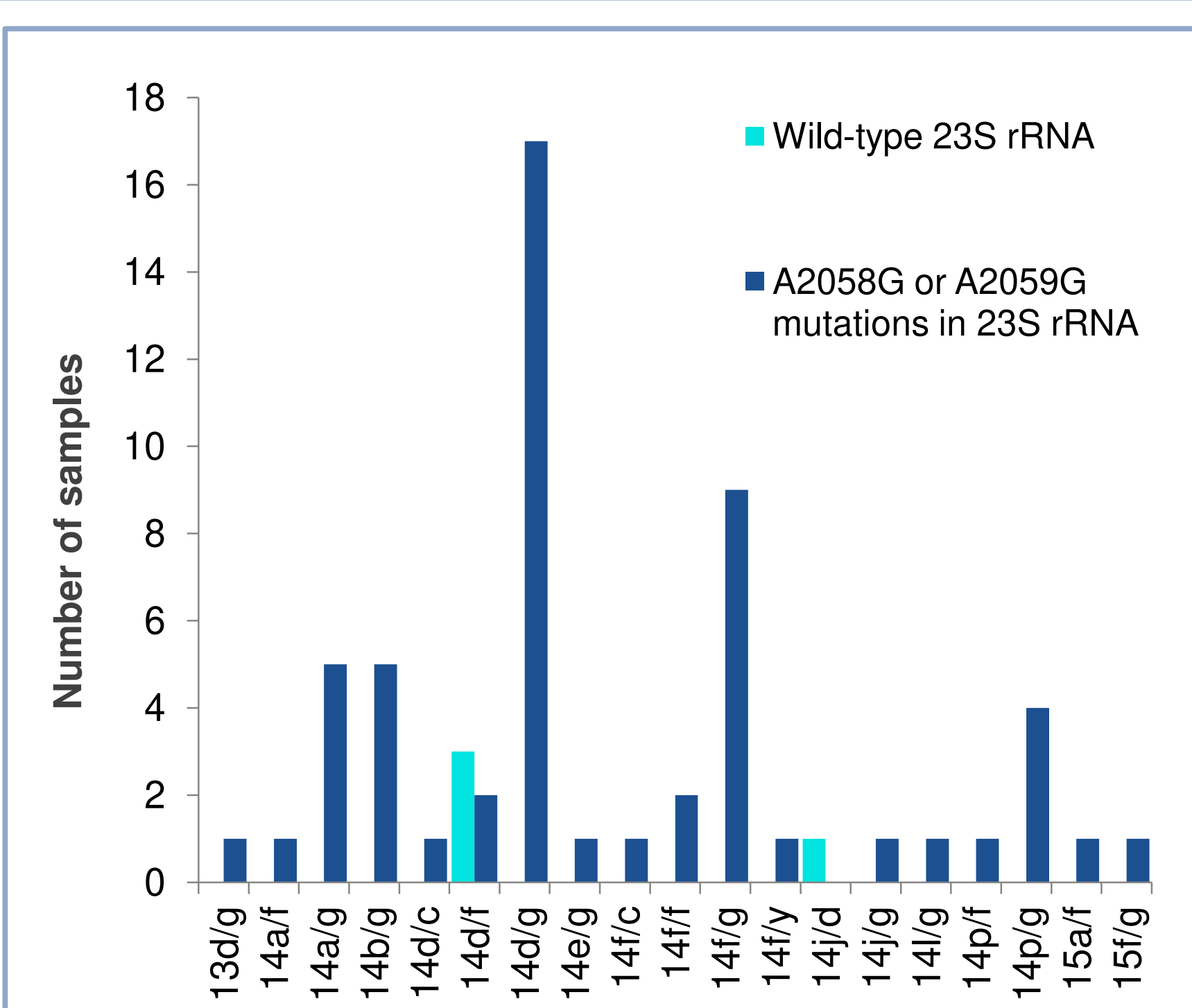

Figure 1. The $T$. pallidum strain-types and 23S rRNA characterisation

\section{CONCLUSIONS}

- To our knowledge this is the first work in Spain describing the genetic macrolide resistance profile of $T$. pallidum. The high frequency $(94 \%)$ of the $23 \mathrm{~S}$ rRNA mutations is similar to the prevalence found in other countries and might be due to the extended use of this family of antibiotics for other STIs treatment.

- The most frequent strain-types showed a genetic resistant profile. In our study, the strain-type $14 \mathrm{~d} / \mathrm{f}$ was identified in only 5 cases, however, in other countries has been described as the most prevalent strain-type. We curiously found that this straintype particularly had lower mutation rates compared to other strain-types.

- Consequently, macrolides are not a real alternative as a second-line antibiotic treatment of syphilis in Barcelona, Spain. 